\title{
What do case reports have to do with population health?
}

\section{Gregory M. Caputo*}

Department of Medicine, Penn State Milton S. Hershey Medical Group, USA

The term "population health" is used in many different ways $[1,2]$. At Penn State Health System, "population health improvement" refers to the measureable improvement in health care value for a well-defined population of individuals. Health care value is defined as better clinical outcomes and patient/family experiences, provided at lower cost. These are the elements of the Institute for Healthcare Improvement's Triple Aim. Individual case reports and reviews such as those published in Clinical Case Reports and Reviews typically describe illustrative, educational, and unusual manifestations of relatively common conditions, or frankly uncommon or rare diseases. What is the link between population health and individual case reports? Is the relationship closer than it seems?

It may appear that there is a dichotomy between individual patient care and population health. After all, traditional patient care requires a single-minded focus on a unique individual, while population health improvement addresses the health and risk factors of large groups of individuals. In reality, though, there is a clear and important relationship between individual care and population health. For example, the measured health of a population is obviously derived from the aggregate health parameters of the many component individuals, as determined by personal behavior, genetic predisposition, social circumstances, environmental exposure, and disease burden. Secondly, the careful analysis of data from large populations can be used to inform individual care. Finally, the lessons learned from unique individuals, including those with atypical manifestations, can be helpful to better understand the wide spectrum of health and disease among larger groups.

Thus, there is actually a continuum between the diagnosis and management of unique clinical presentations and the health of populations of individuals [3]. Uncommon or unusual case scenarios typically illustrate the far end of the population health spectrum. System-based interventions must always be personalized to account for individual preferences, beliefs, values, and clinical situations [4]. Thus, the case report or series may alert us to the possibility that system-based tools might fail to identify or meet the unique needs of individuals with unusual conditions or presentations. For example, an EMR reminder might fail to alert the clinician to the opportunity for better care for the patient with an atypical presentation. Or an unusual syndrome may not be well defined by an existing diagnosis code, and thus the patient may not be accurately identified as part of a larger population of people with that condition.

Like many large regional health systems, Penn State Health has increased its focus on population health improvement. We've grown in our commitment to integrate clinical infrastructure and processes across our region, increase our core capabilities to stratify risk for important outcomes, implement system-based improvements such as electronic medical record (EMR)-based clinical registries and decision support, and strengthen innovative team-based care models.
We typically define sub-populations by age, condition, or geographic region and have learned to avoid reliance on any single source of clinical data - EMR, insurance claims, patient surveys, etc. As part of a recent condition-defined population-based quality improvement project on stroke prevention in atrial fibrillation patients, over 2,000 individual charts were reviewed. In doing so, a close-up, detailed view emerged of the individual care that ultimately aggregates into population-based clinical outcomes. In some cases, the potential benefit of identifying examples of relatively uncommon scenarios or presentations were evident. For example, where does the asymptomatic individual with a high calculated risk of stroke due to age, hypertension, and diabetes, whose sole documented atrial fibrillation episode was during aortic valve replacement surgery, truly fit into the population of purportedly high-risk patients? What might be learned from a careful descriptive review of a multi-center series of such patients?

In evaluating system-based interventions that can improve the health of our regional population, we are increasingly aware of patients who "don't fit the mold" because of unique or unusual manifestations. An excellent recent case report in this journal illustrates this point [3]. A 45-year old woman presented with post-infectious, ascending sensory symptoms which was diagnosed as Acute Disseminated Encephalomyelitis (ADEM) and successfully treatment was implemented. The differential diagnosis of ADEM symptoms is broad, and the condition may be initially misdiagnosed as one of the somewhat more common conditions such as Guillan-Barre Syndrome. There are obvious implications of incorrect diagnosis for an individual patient. Perhaps less obvious are the implications for developing systembased approaches that may improve management of individuals and populations. For example, an EMR-based clinical decision support (CDS) intervention could potentially improve early consideration of ADEM in similar patients. General experience with EMR-derived data suggests that more precise documentation and diagnosis coding among patients with uncommon clinical manifestations could potentially have beneficial effects on the care of individual patients and bring about more accurate and complete description of the target population.

I encourage authors who submit manuscripts to Clinical Case Reports and Reviews to consider commenting on their report's implications for population health improvement and system-based care interventions, when applicable. How might their patient have been identified earlier through system-based interventions? How might population-based knowledge improve care for people like the one described in their manuscript? This approach may lead the readership

Correspondence to: Gregory M. Caputo, Department of Medicine, Penn State Milton S. Hershey Medical Group, USA, Tel: 717-531-1322; E-mail: gcaputo@pennstatehealth.psu.edu

Received: April 06, 2017; Accepted: April 24, 2017; Published: April 27, 2017 
to expand their appreciation of the lessons from case reports, and to better understand the relationship of these unique individuals to the health of our broader population.

\section{References}

1. Gourevitch MN (2014) Population Health and the Academic Medical Center: The Time Is Right. Acad Med 89: 544-9.[Crossref]
2. Kindig D (2015) What Are We Talking About When We Talk About Population Health? Health Affairs.

3. Roshanisefat H, Henderson B, Press, R (2016) An unusual Guillain-Barré Syndrome mimic: a case of Spinal ADEM and review of the literature. Clin Case Rep Rev 2.

4. Jette N, Choi H (2013) Applying evidence to patient care: From population health to individual patient values. Epilepsy \& Behavior 26.

Copyright: $₫ 2017$ Caputo GM. This is an open-access article distributed under the terms of the Creative Commons Attribution License, which permits unrestricted use, distribution, and reproduction in any medium, provided the original author and source are credited. 